\title{
Estimating Contrail Climate Effects From Satellite Data
}

\author{
Patrick Minnis ${ }^{1}$ \\ NASA Langley Research Center, Hampton, VA, 23681
}

David P. Duda ${ }^{2}$, Rabindra Palikonda ${ }^{3}$, Sarah T. Bedka ${ }^{4}$, Robyn Boeke ${ }^{5}$, Konstantin Khlopenkov ${ }^{6}$, Thad $\mathrm{Chee}^{7}$, and Kristopher T. Bedka ${ }^{8}$

Science Systems and Applications, Inc., Hampton, VA, 23681

\begin{abstract}
An automated contrail detection algorithm (CDA) is developed to exploit six of the infrared channels on the 1-km MODerate-resolution Imaging Spectroradiometer (MODIS) on the Terra and Aqua satellites. The CDA is refined and balanced using visual error analysis. It is applied to MODIS data taken by Terra and Aqua over the United States during 2006 and 2008. The results are consistent with flight track data, but differ markedly from earlier analyses. Contrail coverage is a factor of 4 less than other retrievals and the retrieved contrail optical depths and radiative forcing are smaller by $\sim 30 \%$. The discrepancies appear to be due to the inability to detect wider, older contrails that comprise a significant amount of the contrail coverage. An example of applying the algorithm to MODIS data over the entire Northern Hemisphere is also presented. Overestimates of contrail coverage are apparent in some tropical regions. Methods for improving the algorithm are discussed and are to be implemented before analyzing large amounts of Northern Hemisphere data. The results should be valuable for guiding and validating climate models seeking to account for aviation effects on climate.
\end{abstract}

\section{Nomenclature}

$\begin{array}{ll}\text { ACCRI } & =\text { Aviation Climate Change Research Initiative } \\ \text { AVHRR } & =\text { Advanced Very High Resolution Radiometer } \\ \mathrm{BT} & =\text { brightness temperature } \\ \mathrm{BTD} & =\text { brightness temperature difference } \\ B T D i & =\text { brightness temperature difference for wavelength pair } i \\ \mathrm{CDA} & =\text { contrail detection algorithm } \\ \mathrm{COD} & =\text { contrail optical depth } \\ \mathrm{CONUS} & =\text { contiguous United States } \\ \mathrm{DC} & =\text { detection confidence } \\ i & =\text { wavelength index } \\ \text { MERRA } & =\text { Modern Era Retrospective-analysis for Research and Applications } \\ \text { MODIS } & =\text { MODerate-resolution Imaging Spectroradiometer } \\ N & =\text { normalized image sum } \\ \mathrm{NCLRF} & =\text { normalized contrail longwave radiative forcing } \\ N i & =\text { normalized BT or BTD image for wavelength or pair index } i \\ \mathrm{NH} & =\text { Northern Hemisphere } \\ \mathrm{RH} & =\text { relative humidity } \\ S T D i & =\text { local standard deviation of BT or BTD for wavelength } i \\ T i & =\text { brightness temperature for wavelength } i\end{array}$

${ }^{1}$ Senior Research Scientist, Science Directorate, NASA Langley Research Center, Mailstop 420, Hampton, VA, 23681, Member AIAA.

${ }^{2-8}$ Research scientist 


\section{Introduction}

$\mathrm{C}$ LOUDS are an important component of the atmospheric system primarily because of their influence on the radiation budget and the distribution of precipitation. Changes in cloud cover alter the radiation budget and the hydrological cycle and, hence, the climate. Cirrus clouds are generally optically thin and tend to cause a warming of the Earth-atmosphere system, especially when they occur over warm surfaces. Contrails are aircraft-generated cirrus clouds that often form in the absence of other cirrus clouds in ice-supersaturated conditions at temperatures less than $-39^{\circ} \mathrm{C}$. Since such conditions are relatively common in the upper troposphere ${ }^{1,2}$, contrails can develop into relatively long-lived cirrus clouds ${ }^{3}$. Thus, they add to the naturally occurring cloud cover in air traffic corridors and, hence, could be climatically important.

Surface observations suggest that contrails have increased cirrus coverage by roughly $1 \%$ per decade over the Contiguous United States (CONUS) and that decreases in cirrus coverage over Europe expected because of drops in upper tropospheric humidity have been offset by a coincident rise in contrail-generated cirrus clouds ${ }^{4}$. Other studies have shown even larger increases in cirrus coverage over air corridors ${ }^{5,6}$. Model estimates of contrails' impact on the climate vary by, at least, an order of magnitude ${ }^{7}$. Thus, the role of contrails in climate change remains uncertain. Since air traffic is increasing worldwide, contrail coverage is expected to increase during the coming years. Therefore, reducing the uncertainties in the effects of contrails on the climate system is essential to determine whether it is necessary to mitigate their effects.

To reduce those uncertainties, observations and model estimates of contrail properties and their interaction with the atmosphere must be improved. Although estimates of contrail impacts are often based on inferences drawn from surface or satellite observations of cirrus cloud trends, it is preferable to study contrails directly from satellite data using the somewhat unique features of contrails: their typically linear nature and the occurrence of large numbers of relatively small ice crystals in most contrail clouds. The former has been used to estimate contrail coverage through visual inspection of infrared window $(11 \mu \mathrm{m})$ channel imagery ${ }^{8,9}$. While the technique provides some reasonable estimates of contrail coverage, it is fraught with uncertainties due to the non-unique thermal signatures of contrails and clouds and the poor contrast between contrails and clouds. Furthermore, it is labor intensive and highly subjective.

The microphysical properties of contrails provide a means for differentiating contrails from many natural cirrus clouds because the smaller ice crystals in contrails transmit more radiation from the surface at wavelengths around $11 \mu \mathrm{m}$ than at $12 \mu \mathrm{m}$, wavelengths often used for channels on operational meteorological satellite imagers such as the Advanced Very High Resolution Radiometer (AVHRR). This contrail "signature" is manifest in images constructed by subtracting the brightness temperatures (BT) of the split window $(12 \mu \mathrm{m})$ images from their window channel $(11 \mu \mathrm{m})$ counterparts $^{10}$. In these brightness temperature difference (BTD) images, contrails are often evident as relatively bright linear features. The linearity and enhanced contrast in the BTD images allows the development of more objective techniques for identifying contrails and computing properties such as contrail coverage, optical depth, and radiative forcing. Mannstein et al. ${ }^{11}$ exploited these somewhat unique contrail attributes to develop an innovative pattern recognition technique based on the BTD images. Later applications of this contrail detection algorithm (CDA) to various AVHRR datasets yielded estimates of contrail coverage over Europe ${ }^{12}$, the CONUS ${ }^{13}$, the northeastern Pacific ${ }^{14}$ and southeastern Asia ${ }^{15}$.

Although the objective approach for determining contrail coverage significantly decreases the analysis time through automation and minimizes viewer bias, it still suffers from serious detection inefficiencies and from relatively high false alarm rates. Cirrus streamers, especially those associated with convective outflow and subtropical jet streams, often appear linear in the satellite imagery and can be composed of relatively small ice crystals. Thus, they are commonly mistaken by the CDA as contrails. Additionally, background heterogeneity and quasi-linear surface features (e.g, coastlines or rivers) can introduce errors into the CDA results. Statistical methods based on results in air-traffic-free regions ${ }^{12}$ or on subjective image analyses ${ }^{13,14}$ have been used to account for false alarm frequencies that are often as large as or greater than the true detection frequencies, especially over land areas. Detection efficiency, i.e., the probability of a correct positive detection, relative to subjectively determined contrail coverage from AVHRR or other sources varies from 40-90\%. Yet, as indicated by comparisons with surface observations ${ }^{16}$ and model calculations ${ }^{17}$, the subjective analyses are not infallible references; they often miss those contrails that are narrow, optically thin, and $>5 \mathrm{~km}$ in width. The detection efficiencies become even worse as the satellite image resolution decreases. Thus, the automated techniques still require improvement along with careful application and interpretation.

Estimates of contrail optical depth (COD) and radiative forcing have also been estimated for the detected contrails in several of the aforementioned studies. The mean contrail COD over the CONUS ${ }^{13}$ and adjacent Pacific Ocean $^{14}$ was $\sim 0.27$, more than double that found over Europe ${ }^{12}$. The regional differences may be due to differences 
in retrieval approaches or variations in the temperatures and humidities at contrail flight altitudes The estimates of radiative forcing over those regions are difficult to compare because of the different approaches used their derivation.

Persistent contrails, those that can be seen in satellite images, form wherever aircraft exhaust mixes with air having the potential to form natural cirrus clouds. Since satellite images have been analyzed for contrails over only a few portions of the globe, the current record of direct observations of contrails is inadequate, notwithstanding the detection difficulties noted above. This paucity of analyses is, in great measure, due to the lack of global $1-\mathrm{km}$ AVHRR data. With the launching of the Terra and Aqua satellites in 2000 and 2002, respectively, and the operation of their modernized data storage and transmission systems, many years of well-calibrated, relatively high-resolution (1-km), multispectral imager data from the MODerate-resolution Imaging Spectroradiometer (MODIS) are available over the entire Earth. Thus, it is now possible to develop global climatologies of contrails and their properties.

In this paper, a revised CDA is developed to take advantage of some of the additional channels on MODIS and a number of analyses are performed. The new CDA is first applied to MODIS data over the CONUS to determine the coverage and radiative impacts for this region of dense air traffic for two different years. Initial results of a northern hemisphere (NH) analysis are presented and discussed. Finally, a roadmap for a more comprehensive NH analysis is developed in order to obtain contrail radiative forcing and particle size estimates. The results should be valuable for guiding and validating climate model contrail impacts to improve climate predictions for the expected increases in global air traffic.

\section{Method}

The CDA is a modified version of the technique described by Mannstein et al. ${ }^{11}$, which detects linear contrails in multi-spectral thermal infrared (IR) satellite imagery using only two channels $(11 \& 12 \mu \mathrm{m})$ from the AVHRR. This method requires only the brightness temperatures from the IR channels, with no other ancillary data, and can be applied to both day and night scenes. It uses a scene-invariant threshold to detect cloud edges produced by contrails, and 3 binary masks to determine if the detected linear features are truly contrails. However, these masks are not always sufficient to remove all non-edge features. To reduce the number of false positive detections due to lower cloud streets and surface features, we add observations from other IR radiance channels available on the MODIS. The new modified method uses additional masks derived from the added thermal infrared channels to screen out linear cloud features that appear as contrails in the original method.

The modified CDA follows the same overall data flow as the original Mannstein et al. method, but also uses the following BT data to compute various BTD images:

$$
\begin{array}{rl}
T 6.8: 6.8-\mu \mathrm{m} \mathrm{BT} & T 8.6: 8.6-\mu \mathrm{m} \mathrm{BT} \\
T 11: 10.8-\mu \mathrm{m} \mathrm{BT} \quad T 12: 12.0-\mu \mathrm{m} \mathrm{BT} \quad T 13: 13.3-\mu \mathrm{m} \mathrm{BT} \\
B D T 1=T 11-T 12, \\
B T D 2=T 8.6-T 12, \\
B T D 3=T 8.6-T 13, \\
B T D 4=B T D 1+B T D 2 .
\end{array}
$$

Normalized images $N i$ are computed for each $T i$ and $B T D i$ image in the following manner, where $i$ denotes the BT wavelength or wavelength pairs used in a BTD.

$$
N i=(T i-<T i>) /(S T D i)+0.1 \mathrm{~K})
$$

where the brackets indicate averages of Gaussian-smoothed 5x5 pixel array centered on the given pixel and the local standard deviation $S T D i$ is computed for each pixel using the surrounding $5 \times 5$ pixel array.

The sum of the normalized images

$$
N=N 12+N B T D 1+N B T D 2
$$

was then convolved with a line filter of $19 \times 19$ pixels in 16 different directions. The individual connected regions resulting from the filtering are considered as possible contrail objects.

These objects are then compared with 6 binary masks to check for contrails. Duda et al. ${ }^{18}$ describe these masks in some detail Although the first three masks are similar to the original BTD method, they utilize information from two other channels to help eliminate coastlines and improve detection of contrails over opaque low clouds. The last three 

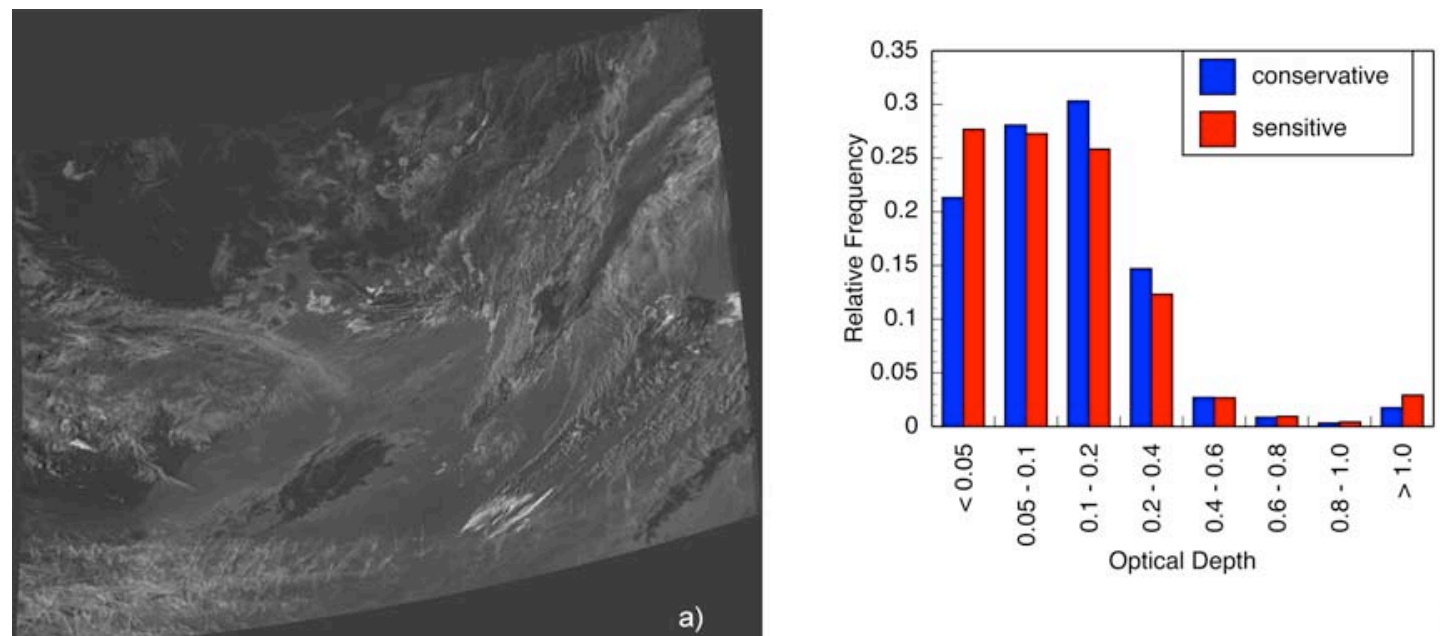

a)

b)
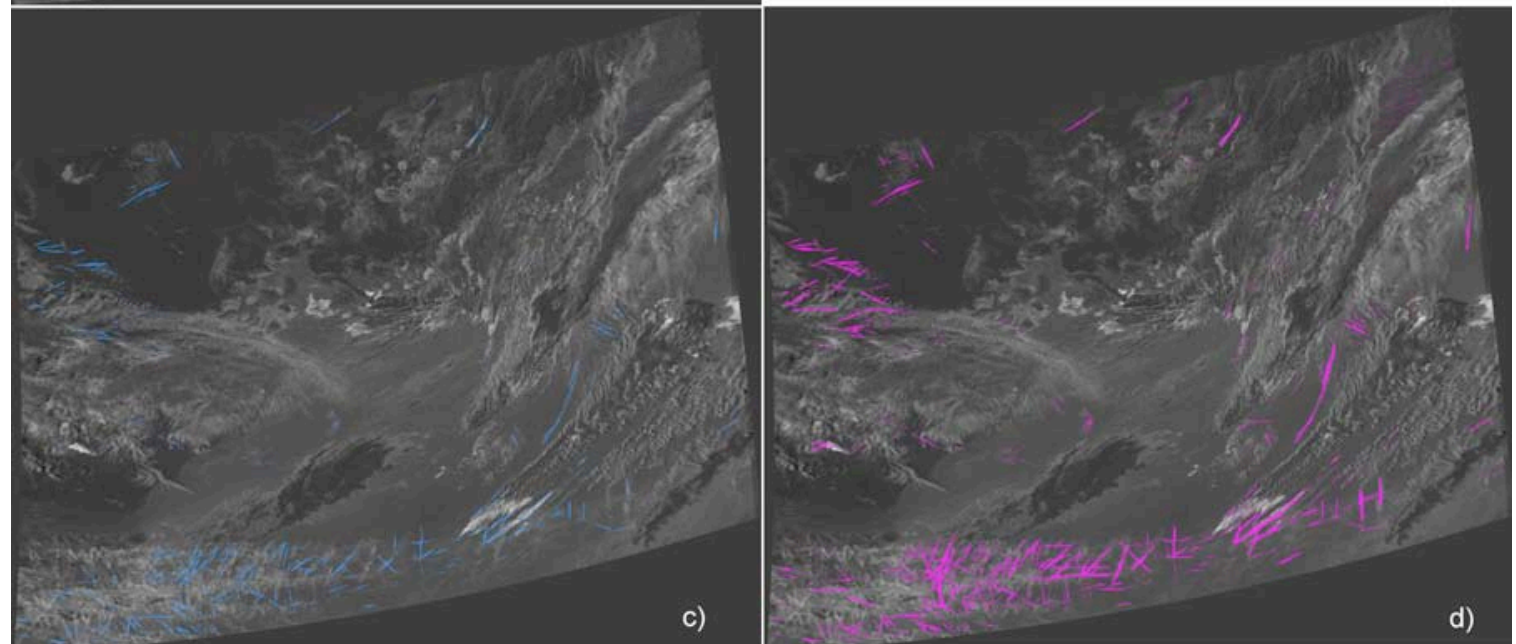

Figure 1. Brightness temperature difference image, contrail masks, and retrieved contrail optical depths from Terra MODIS image taken over eastern CONUS, 1530 UTC, 1 January 2006. (a) BTD1, (b) COD distribution, (c) BTD1 with CDA1 mask, and (d) BTD1 with CDA6 mask.

binary masks are used to reduce the number of false positive detections of lower level cloud streets and other edge features in the IR imagery. They use information from the new channels, mostly in the form of thresholds on gradients in the various constructed images.

By varying the thresholds used in each step, it is possible to develop a very conservative mask that produces few false alarms or a sensitive mask, similar to the original Mannstein CDA, that has a high level of false alarms but also detects more contrails. Six sets of thresholds were established with CDA1 as the most conservative mask and CDA6 as the most sensitive contrail mask. The more sensitive masks also allow the detection of wider contrails. Figure 1 shows an example of the modified CDA applied to Terra MODIS data taken over the eastern USA, 1530 UTC, 1 January 2006. The BTD1 image (Fig. 1a) reveals contrails near the bottom of the image and another patch of contrails in the upper left of the image. CDA1 detects many of the contrails seen in the BDT1 image (Fig. 1c), while CDA6 (Fig. 1d) detects wider contrails, more contrails altogether, and some more questionable contrails.

After detecting the contrails, the COD and the longwave radiative forcing of the contrails are determined using the same techniques employed by Palikonda et al. ${ }^{13}$, except that the contrail temperature is assumed to be $220 \mathrm{~K}$ instead of $224 \mathrm{~K}$. Examples of the COD distributions for the image in Fig. 1a are shown in Fig. 1b. The conservative mask tends to detect a greater proportion of contrails having COD between 0.1 and 0.4 , while contrails with COD < 0.1 are more common for the sensitive mask. Overall, the difference in the average CODs is only 0.02 .

To develop a climatological database of contrail coverage and optical properties, it is necessary to determine which CDA is best. It is assumed here that the optimal CDA is the one that produces the smallest bias, while minimizing the false alarm rate. Thus, the optimal method will produce roughly the same amount of false contrail 


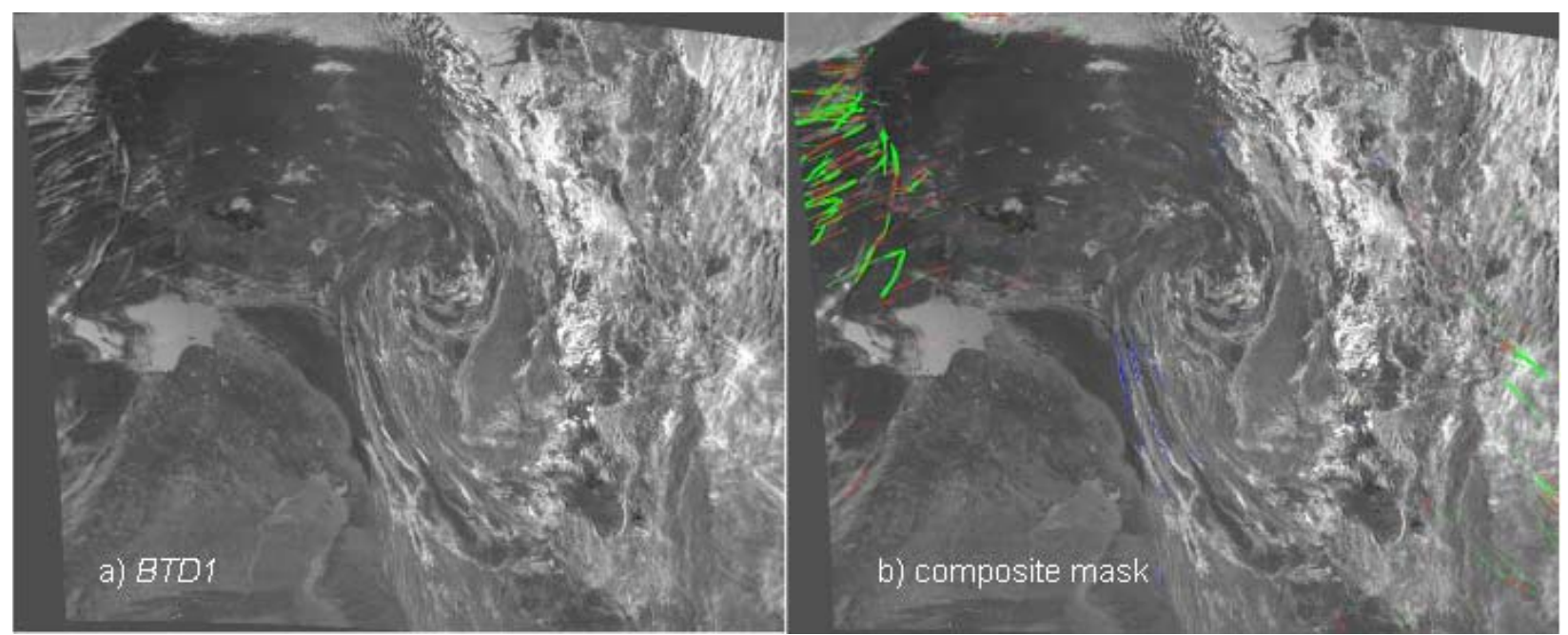

Figure 2. Terra MODIS image, 1605 UTC, 1 April 2006 over CONUS. (a) BTD1, (b) BTD1 with composite mask from 4 analysts showing CDA1 contrails in red (confirmed by analysts) and blue (rejected by analysts) and contrails added by the analysts in green.

and missed contrail pixels. To determine this optimal technique, a subjective error analysis was performed using an updated version of the interactive program developed by Minnis et al. ${ }^{14}$ The CDAs were first applied to sets of 20 daytime and 20 nighttime MODIS images covering the four seasonal months (January, April, July, and October) over CONUS. The results were evaluated interactively by 4 different analysts to estimate the number of false detections, missed contrails, and positive contrail detections. A composite mask for each image was determined from the four results. The results from CDAs 1-6 were evaluated with this composite mask. Figure 2 shows an example of the composite visual analysis. Figure 2a shows a typical BTD1 image used to find contrails visually, while Fig. $2 \mathrm{~b}$ highlights the pixels identified by the CDA (red), as well as the pixels determined by the analyst to be additional contrails missed by the CDA (green). Pixels expected to be false positives are shown in blue near the center of the image. In this instance, the number of missed pixels far exceeded the false alarms.

The ultimate goal of the CDA is to produce an unbiased estimates of linear contrail coverage, and contrail microphysical properties and radiative forcing. To meet that objective, the 40 test images were used to select the CDA that generated as many false positive detections as missed contrails. Overall, it was found that CDA1 and CDA3 produced the smallest biases for night and day, respectively. This day-night difference in sensitivities is due to the change in the background radiances from day-to-night. Over land, BTD1 can change significantly over the course of the diurnal cycle for clear scenes.

While it is assumed that the analysts' subjective assessments constitute the "truth" set for the CDA selection, it is apparent in viewing many of the images that defining a linear contrail is not always straightforward. This difficulty arises primarily from the presence of older contrails that have spread significantly. Figure 3 shows an example of scenes containing older contrails amongst younger ones. The CDA detected several relatively wide contrails in the lower right portion of the image that were confirmed by the analysts (red), but it missed a few unambiguously identified by the analysts (green), while apparently incorrectly identifying one that was orthogonal to the others. Close inspection of the imagery suggests that the clouds in that vicinity are very likely older contrails that have lost some of their distinct contrail "signatures", having diffused, overlapped, and grown in particle size. This evolution ${ }^{19}$ is common, especially in contrail outbreaks ${ }^{20}$. Such outbreaks may be responsible for much of the excess cloudiness due to contrails, but are difficult to quantify with automated methods and, hence, are analyzed manually ${ }^{20}$. The ambiguity resulting from the older and overlapping contrails in such outbreaks should be considered when defining the extent of linear contrails. Since they do not appear to be included in the current reference dataset, it is likely that the truth set from the analysts is an underestimate and the corresponding CDA results will also underestimate the true contrail coverage.

\section{Results and Discussion}

This section presents the preliminary results for analyses of data taken over the CONUS and NH. Possible improvements are also discussed. 


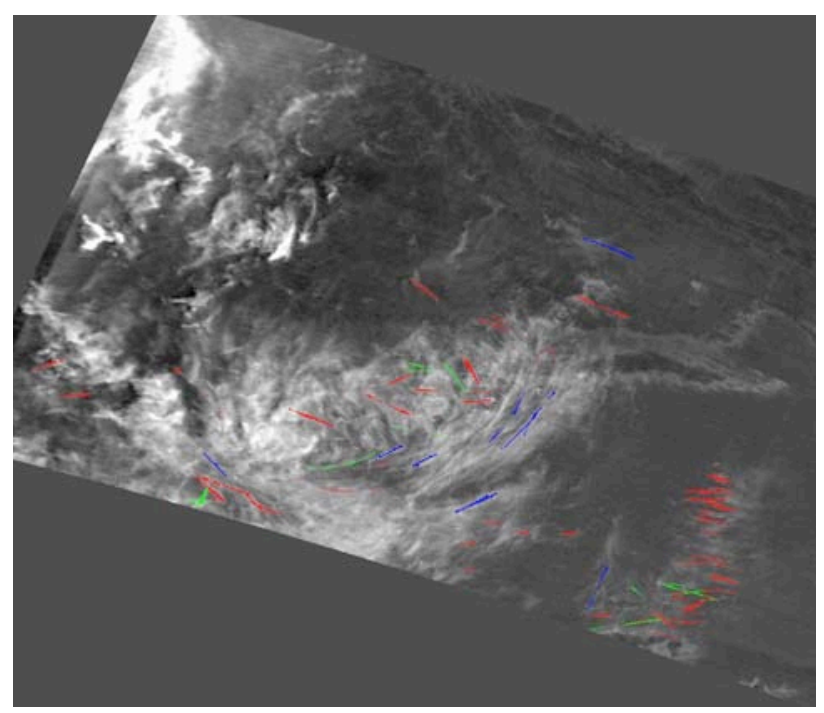

Figure 3. Terra MODIS image, 0312 UTC, 2 April 2006 over CONUS showing BTD1 with composite mask. Color codes are same as those in Fig. 2.

\section{A. CONUS Results}

The optimal algorithms were used to analyze 2006 and 2008 Terra and 2006 Aqua MODIS imagery taken over the CONUS. Figure 4 shows the Terra results for daytime 2006.The greatest and least contrail coverage occur during winter (DJF) and summer (JJA), respectively. During winter, the densest contrail coverage is seen over the Mississippi valley and the southeastern CONUS. During summer, the maxima shift more to the north. During spring and fall, the peak coverage is seen over the central Great Plains. The nocturnal coverage (not shown) drops by a factor of two. The seasonal variability is similar to that seen in earlier studies ${ }^{13,21}$. During 2008 (Fig. 5b), the contrail coverage is distributed differently than during 2006 (Fig. 5a) indicating that meteorology, more so than air traffic, governs the contrail coverage over CONUS since the air traffic patterns do not shift significantly from year to year.

Figure 6 shows the 12-month average contrail coverage for 2006 derived from Aqua data taken at 0130 and 1430 LT. The daytime coverage (Fig. 6a) shows relative maxima over Iowa, Kansas, and the Gulf Mexico, around Lakes Erie and Superior, and off the coasts of Maine, Nova Scotia, and Oregon. The nocturnal peak shifts a little to the southwest over the common borders of Missouri, Iowa, Kansas, and Nebraska, while other relative maxima are seen over western Colorado, Mississippi, the Gulf of California, and off of Nova Scotia. The upper-tropospheric air traffic data shown in Fig. $6 \mathrm{~d}$ are for 10 September $2001^{22}$ and should be typical of the distributions over CONUS. In many respects, the patterns are in agreement but differ significantly in some areas. The relative maxima over the Bahamas, the Gulf of Mexico, central California, Missouri, Iowa, New Mexico, Arkansas, and other areas fit the pattern of air traffic. However, fewer contrails are detected over northern Florida, northern California, Oregon, and the states surrounding Kentucky than expected given the density of air traffic. Relative to the air traffic, it appears that more contrails are detected over water surfaces than over land and fewer contrails are seen over mountainous areas (e.g., Great Smoky Mountains over North Carolina and Tennessee, Rocky Mountain West).

The relative minima over mountainous areas may be due to several factors. A factor recognized by Mannstein et al. ${ }^{11}$ is thermal heterogeneity of the background, which increases with elevation and terrain height variability. Thus, it is more difficult to distinguish contrail pixels from the background pixels in mountainous areas. Conversely, over water surfaces, heterogeneity is minimized and detection efficiency should be maximized. Circulation perturbations by mountains may also have some impact on the formation of contrails long enough to be detected in the satellite imagery. The occurrence of the maximum coverage over Iowa instead of the heaviest traffic areas of the midwestern CONUS may be due to saturation effects. As noted earlier, the overlapping and spread of contrails can diminish the detection efficiency. Carleton et al. ${ }^{20}$ report that contrail outbreaks occur twice as often over the Midwest than over any other CONUS region. Thus, the bulk of contrail coverage over this heavy air traffic region may be missed because too many contrails of various ages occur together and are difficult to separate in an automated fashion.

Overall, the Terra CONUS contrail coverage during 2008 is $0.15 \%$ compared to $0.16 \%$ during 2006 . On average, the 2006 Aqua analyses yielded a mean CONUS contrail coverage of $0.13 \%, 0.15 \%$ during the day and $0.11 \%$ at night. These values are roughly a factor of 4 smaller than the CONUS averages determined from AVHRR data taken during $2001^{13}$ 

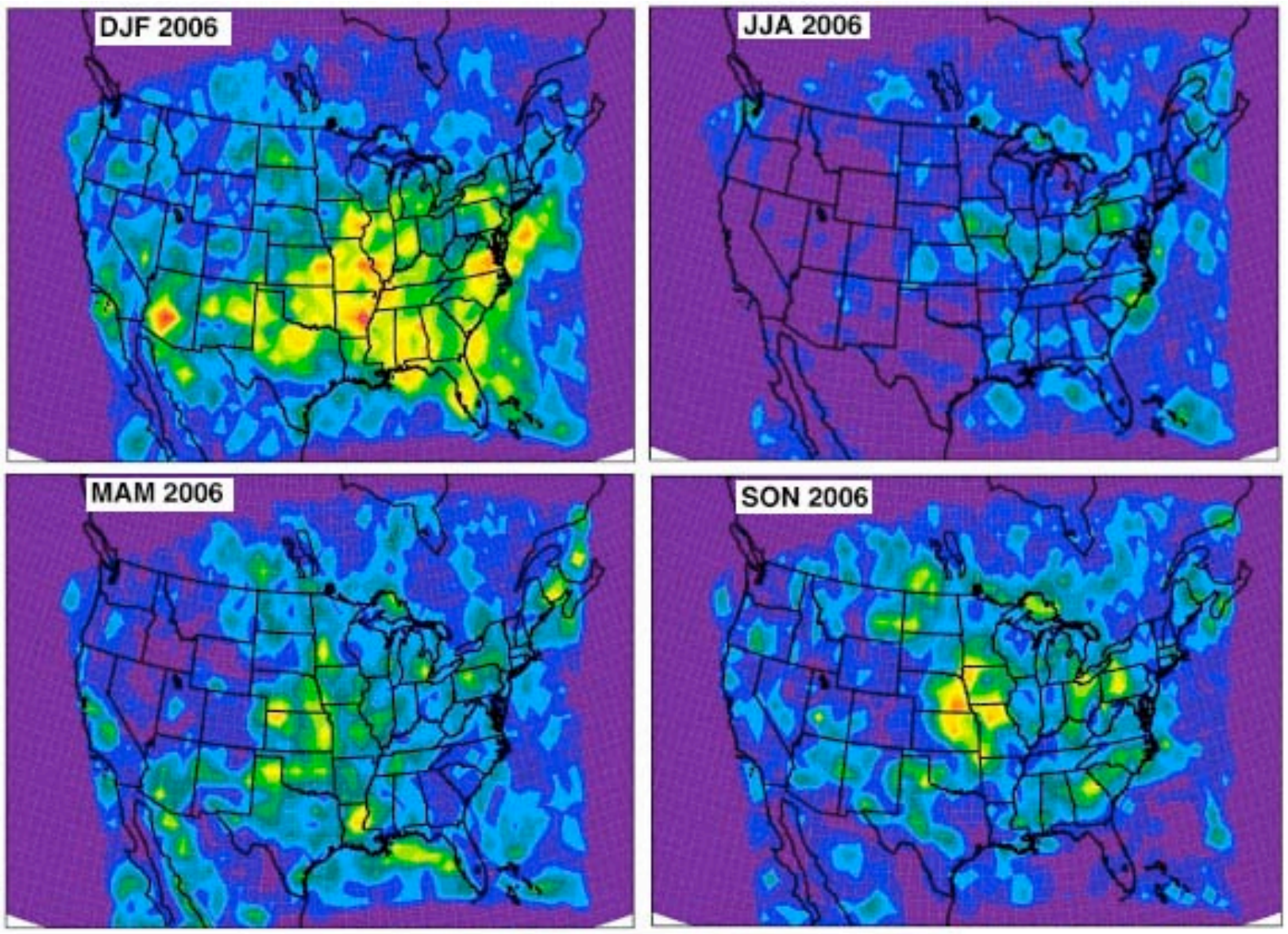

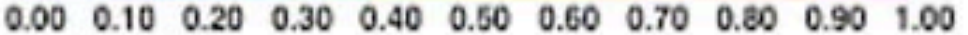

Figure 4. Seasonal mean daytime contrail coverage from Terra MODIS during 2006 using CDA3.
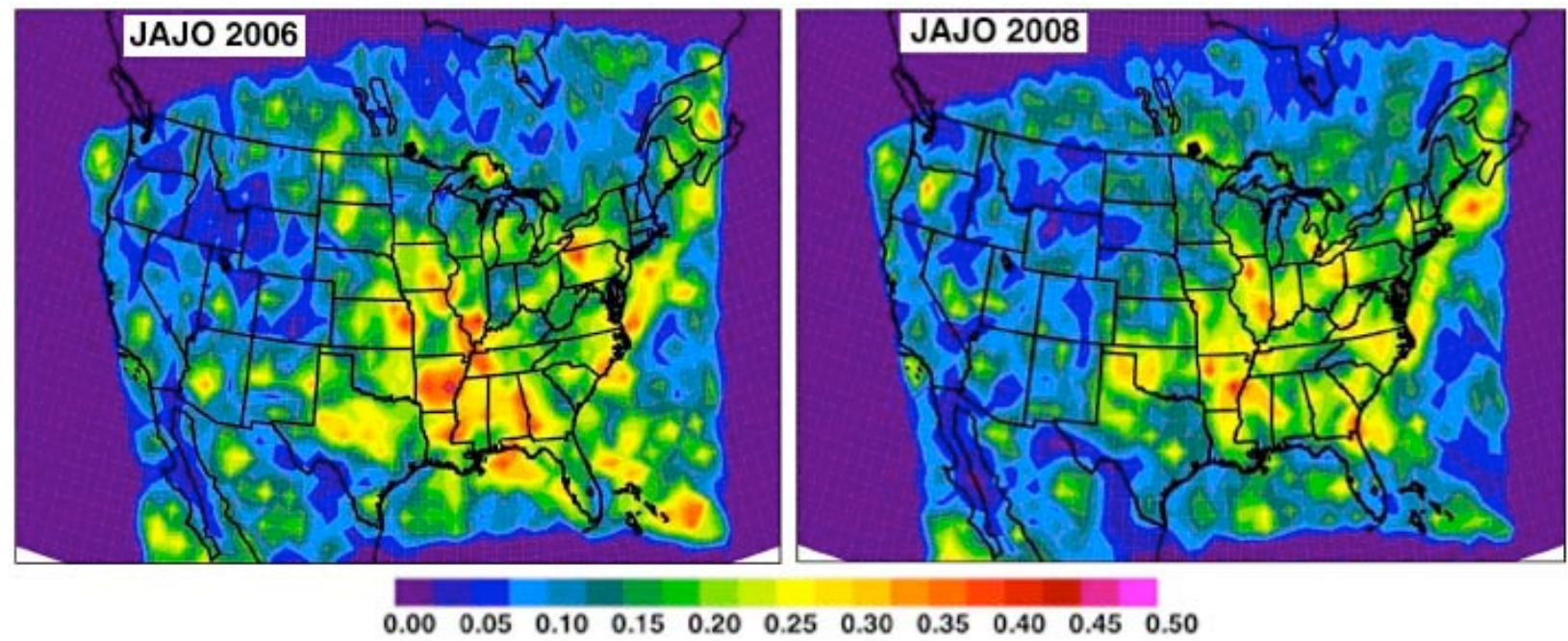

Figure 5. Mid-season monthly averages of contrail coverage, day and night, determined from Terra MODIS data during 2006 (left) and 2008 (right) over CONUS using CDA3. 

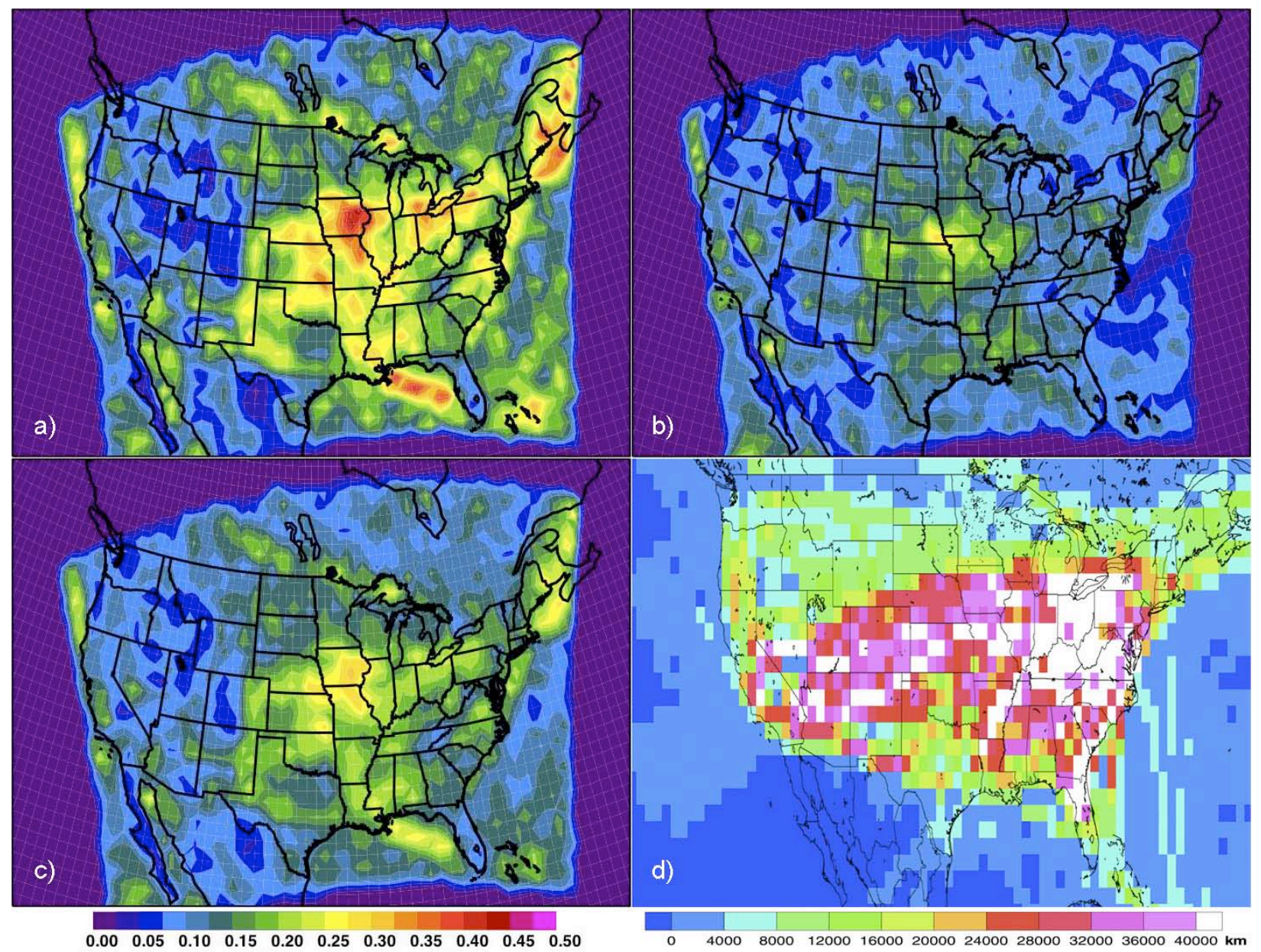

Figure 6. Mean contrail coverage from Aqua MODIS data, 2006 and 2001 air traffic. Contrail coverage during (a) daytime only, (b) night only, and (c) night + day. (d) Mean 24-hr flight distance above 25,000 ft per $1^{\circ}$ region.

Figure 7 compares the Terra 2006 seasonal month COD distributions to those from the NOAA-16 AVHRR results of Palikonda et al. ${ }^{13}$ The peak of the histogram is between 0.2 and 0.4 for the AVHRR results compared to the Terra values. In a relative sense, many more optically thin contrails were detected in the MODIS data than in the AVHRR retrievals. Nearly $40 \%$ of the Terra contrails have COD $<0.2$ compared to $\sim 23 \%$ for NOAA- 16 . The average Terra COD was 0.18 during 2006 and 2008. These new values are almost 0.10 smaller than found in earlier studies $^{13,14}$, but are closer to those found over Europe ${ }^{12}$. Either the older analyses yielded overestimates of COD and contrail coverage, or these new preliminary analyses are too conservative, and/or detect a greater proportion of optically thin contrails, reducing the average COD. As noted earlier, the CDA optimally matches the analysts' amounts, but probably underestimates the true value because it misses the wider contrails, which may be optically thicker than the narrow ones. The average Aqua COD of 0.20 was slightly greater during the day $(0.21)$ than at night (0.19). The normalized 2006 contrail longwave radiative forcing (NCLRF) from Terra and Aqua are 10.2 and 11.0 $\mathrm{Wm}^{-2}$, respectively. NCLRF differed by only $0.4 \mathrm{Wm}^{-2}$ between day and night. The COD was slightly greater during winter than in summer, but the NCLRF was greatest during summer due to the warmer background. Overall, the MODIS COD and NCLRF are 25 and 35\% less than found from the 2001 AVHRR results.

\section{B. Preliminary NH Results}

The optimal CDAs were applied to Terra MODIS data taken 15 October 2006 over all of the Northern Hemisphere. The results are plotted in Fig. 8 along humidity and flight distance density information. The CDA (Fig. 8a) detects a considerable amount of contrail coverage in the tropics including the southern Sahara Desert. Contrails also occur over the Arctic and, over the CONUS, a considerable amount occurs over Oregon, Nebraska, Minnesota, central Texas, and a few other areas. A line extending from west Texas across Mexico blossoms into a wider area over the 

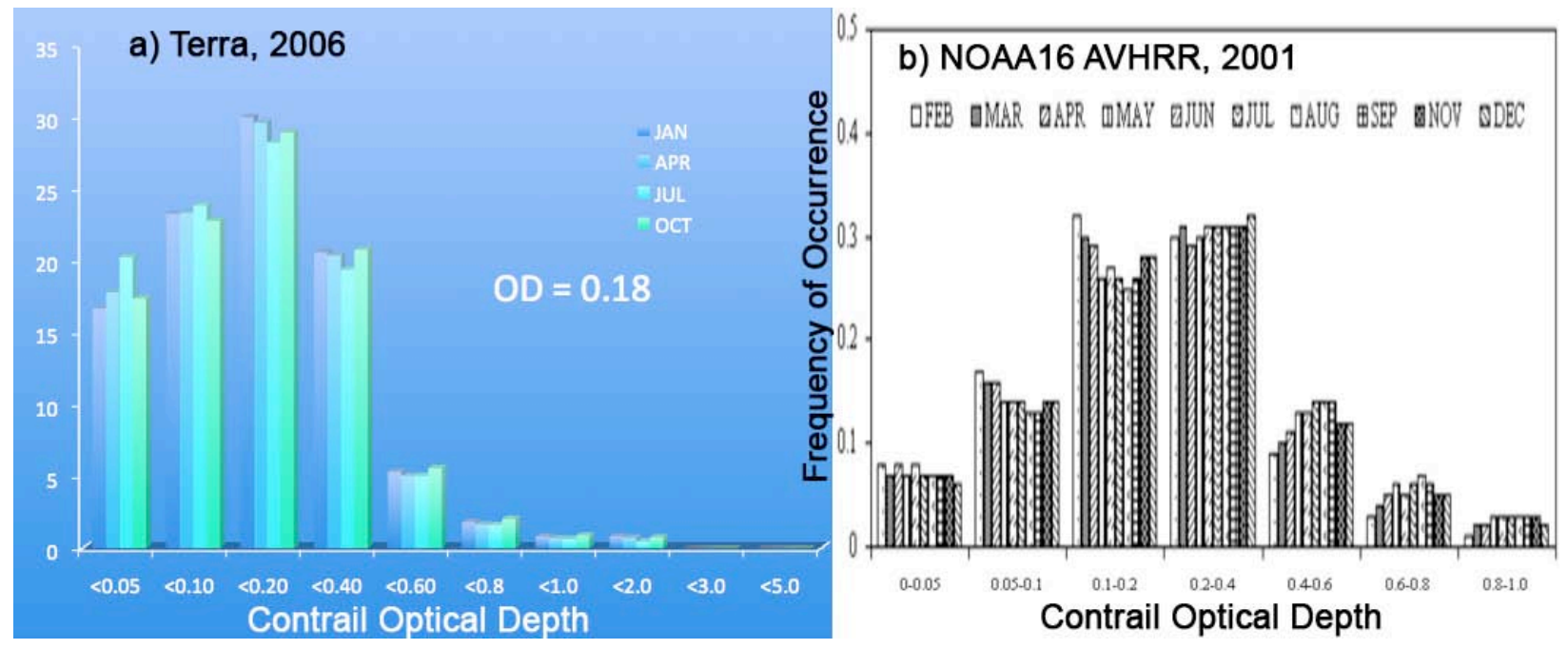

Figure 7. Probability distributions of COD from two different CONUS datasets.

Pacific (Fig. 8a). The flight densities are plotted in Fig. $8 \mathrm{~b}$ for the 3 hours before the Terra overpass and in Fig. $78 \mathrm{~d}$ for the entire day. The densities are taken from the Aviation Climate Change Research Initiative (ACCRI) flight waypoint dataset (see Acknowledgments) and expressed in the number of 7.5-km flight segments above 23,500 ft per $1^{\circ}$ region. Assuming a contrail only retains its features for less than 3 hours, the densities in Fig. $8 \mathrm{~b}$ should correspond most closely with the contrail patterns seen in Fig. 8a. In a number of instances, the flight tracks and contrails coincide (e.g., central Canada, Oregon, North Atlantic, around the Iberian Peninsula). If the flight tracks for the entire day are considered, more of the contrails line up with the flight tracks.

There are many areas where flights occur and no contrails are detected, and vice versa. The former case is often explainable by the humidity fields in the upper troposphere. Figure 8c plots the 24 -h mean relative humidity (RH) with respect to liquid water at an atmospheric pressure of $300 \mathrm{hPa}$. The means were computed from the Modern Era Retrospective-analysis for Research and Applications ${ }^{23}$ (MERRA) analyses. For example, despite the large number of flight segments west of southern California, no contrails are detected. The RH data in Fig. 8c indicate that those flight tracks passed through very dry, unsaturated air (ice supersaturation is roughly at $\mathrm{RH}=70 \%$ ) and no contrails could form. Further south in Pacific, there are few flight tracks and significant contrail coverage coinciding with large values of RH. Other examples can be seen between the Equator and $20^{\circ} \mathrm{N}$. Over those areas, the CDA is likely picking up too many contrails in the naturally occurring cirrus clouds. The CDA was only evaluated over the CONUS, where fewer cirrus streaks are likely to occur than over the tropics. Examination of the images for some of the areas having contrails and no flight data revealed that the cirrus clouds were streaky and could be mistaken for contrails in the CDA and, sometimes, in visual inspection.

\section{Potential Improvements}

The initial results from the analysis of the MODIS data have highlighted many of the difficulties associated with detecting and retrieving contrail properties over the globe using an automated satellite analysis technique. While it is clear that many contrails, especially thicker, older ones, are missed using the CDAs developed here, the number of clouds mistaken as contrails has been reduced significantly relative to previous versions, except in the tropics. Thus, to obtain a more comprehensive and reliable record, a number of improvements are needed.

Better detection of wider contrails and those occurring in overlapped conditions is needed. But it must be balanced against the requirement of minimal false positives. One approach is to loosen the thresholds applied in the CDA and expand the allowable width in the retrieval. This can be done to some extent by determining a set of radiances characteristic of the more certain detected contrails and performing a search of the contrail surroundings for pixels having similar radiances. Initial tests of this approach show some promise, but inevitably some false positives will arise. The lengths to which this approach can be taken will depend on the false alarm rate that is generated. The effects of background heterogeneity can also be minimized by applying statistical corrections ${ }^{11,12}$ to the monthly means.

To reduce the occurrence of false contrail detections such as those over the equatorial Pacific, adjustment of the multispectral thresholds and comparisons with flight track data can be useful. The waypoint data from the 2006 ACCRI flight track database can be used in conjunction with the MERRA analyses to realistically simulate contrails 

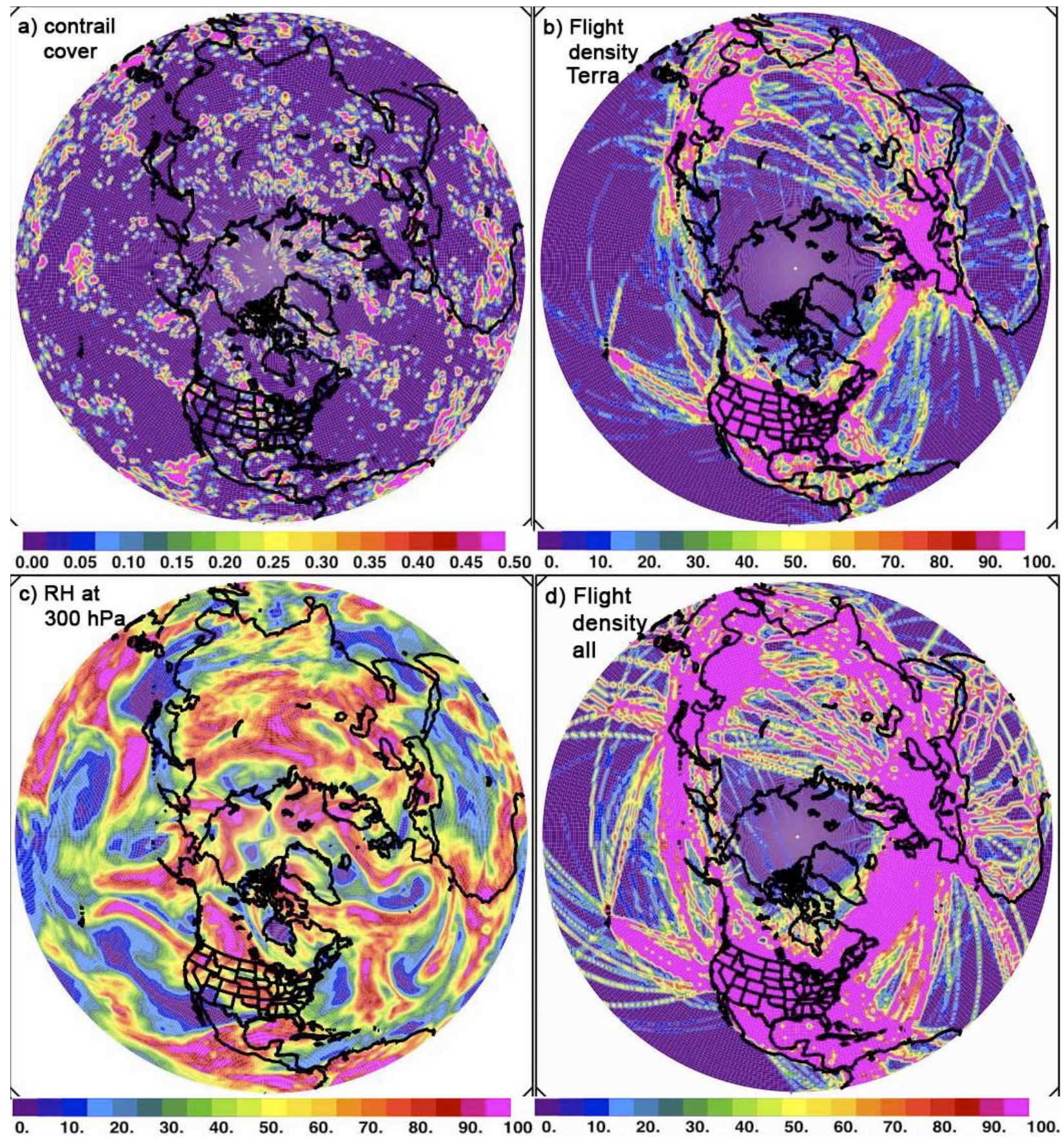

Figure 8. Contrail, flight, and humidity data for 15 October 2006. (a) NH contrail coverage (\%) from Terra MODIS; (b) Number of $7.5 \mathrm{~km}$ flight legs above 23,500 ft up to 3 hours before Terra overpass. Values exceeding 100 are shown in magenta; (c) relative humidity (\%) at $300 \mathrm{hPa}$; (d) same as (c), except for entire day.

that can be used to effect such a comparison. This is accomplished by advecting the flight tracks with the flight-level winds, spreading the track using a diffusion rate, and allowing for an error in the wind fields and location of the aircraft. The orientation and proximity of the contrails detected with the CDA can then be compared with the location and orientation of the simulated contrails.

Figure 9 shows an example after such a comparison. Each contrail pixel is assigned a detection confidence (DC) value between 0 and 100 based on how well it corresponds to a set of simulated contrails determined from the ACCRI flight database and MERRA analyses. The cooler colors indicate a high level of confidence while the 


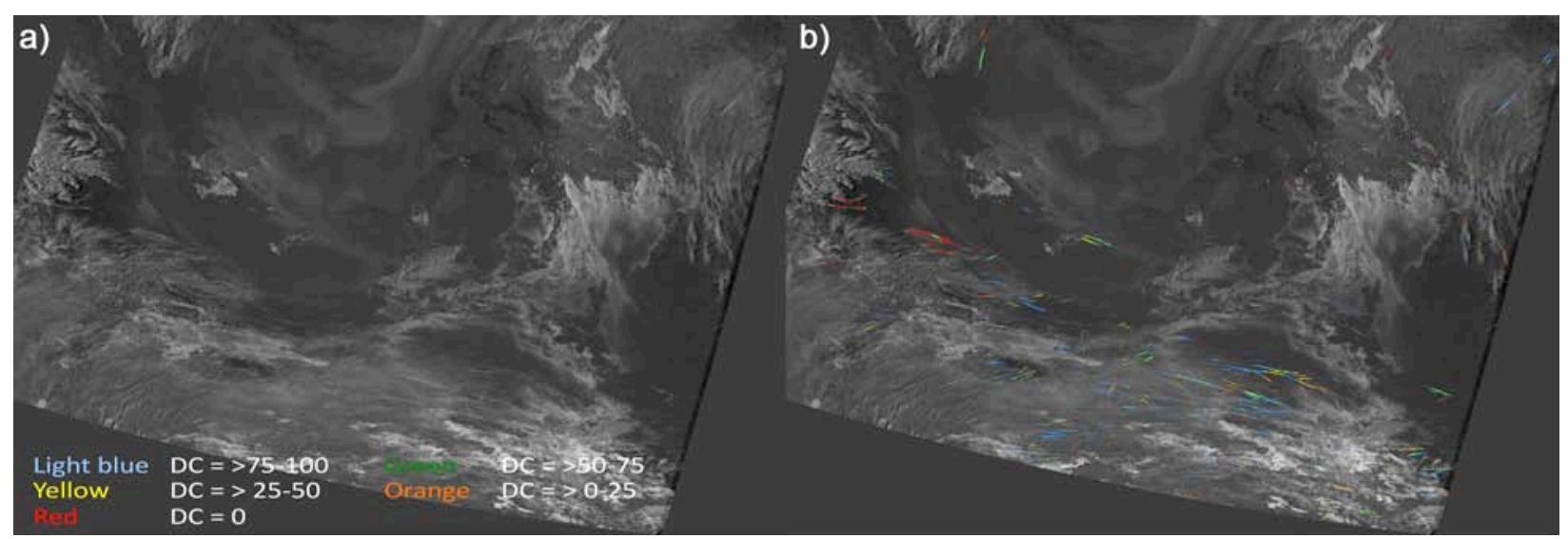

Figure 9. Example of using aircraft waypoint data to assign confidence to a given contrail retrieval, 1705 UTC, 1 January 2006 over northern Great Plains (a) Terra MODIS BTD1, (b) CDA detection confidence (DC).

warmer colors indicate poor matching with the available flight information. The red colors primarily occur in the leftmost part of the image while DC is relatively high for the rest of the image. In some cases, a given contrail has both red and green segments. While this prototype approach appears to be useful, it is clear that using the flight track data can be extremely complicated and require further refinement. Nevertheless, it should prove quite valuable in areas where flight densities are low (e.g., the tropics). Further testing is underway.

Other improvements are planned that will be included with the final algorithm. These include the retrieval of cloud properties for all of the images using established techniques ${ }^{24,25}$, so that contrails can be placed in the context of their surroundings. The additional information will permit the calculation of both shortwave and longwave radiative forcing. Additionally, the flight track data will be used to assign a more accurate temperature to each contrail instead of assuming a constant value for all contrails. Whenever the contrails occur above a cloud-free background, the nighttime retrieval method of Minnis et al. ${ }^{25}$ will be used to estimate the effective particle size. This will allow more accurate calculations of COD and radiative forcing.

\section{Conclusion}

A new contrail detection algorithm has been developed by combining additional spectral information with an older two-channel technique. The new method eliminates many surface and low-cloud features that produced false positive detections in the older method. It has been applied to 2 years of MODIS data over the CONUS and detects fewer contrails than the previous techniques but has much reduced false alarm rates. The new CDA finds fewer contrails, but it also finds more contrails with lower optical depths than found with the earlier approach. The CDA apparently misses many of the thicker, wider contrails observed in contrail outbreaks and should be improved to account for those important components of the contrail coverage.

Discrepancies between the new results and those from earlier studies will be analyzed in the future. Additionally, the use of air traffic flight tracks to improve the cloud mask will be further examined. When the final CDA is selected, it will be applied to MODIS data taken over the Northern Hemisphere to enhance our knowledge of contrail coverage and contrail radiative effects.

\section{Acknowledgments}

This research is supported by the Federal Aviation Administration Aviation Climate Change Research Initiative (ACCRI) program, and by funding from the American Recovery and Reinvestment Act (ARRA). The emissions inventories used for this work (cited as "ACCRI" data in the text) were provided by US DOT Volpe Center and are based on data provided by the US Federal Aviation Administration and EUROCONTROL in support of the objectives of the International Civil Aviation Organization Committee on Aviation Environmental Protection $\mathrm{CO}_{2}$ Task Group. Any opinions, findings, and conclusions or recommendations expressed in this material are those of the authors and do not necessarily reflect the views of the US DOT Volpe Center, the US FAA, EUROCONTROL or ICAO. 


\section{References}

${ }^{1}$ Gierens, K., Schumann, U., Helten, M., Smit, H., and Marenco, A. "A distribution law for relative humidity in the upper troposphere and lower stratosphere derived from three years of MOZAIC measurements," Ann. Geophysicae, Vol. 17, 1999, pp. $1218-1226$

${ }^{2}$ Gettelman, A., Collins, W. D., Fetzer, E. J., Eldering, A., Irion, F. W., Duffy, P. B., and Bala, G. "Climatology of upper tropospheric relative humidity from the Atmospheric Infrared Sounder and implications for climate," J. Climate, Vol. 19, 2006, pp. 6104-6121.

${ }^{3}$ Minnis, P., Young, D. F., Nguyen, L., Garber, D. P., Smith, W. L., Jr., and Palikonda, R., "Transformation of contrails into cirrus during SUCCESS," Geophys. Res. Lett., Vol. 25, 1998, pp. 1157-1160.

${ }^{4}$ Minnis, P., Ayers, J. K., Palikonda, R., and Phan, D. N., “Contrails, cirrus trends, and climate,” J. Climate, Vol. 17, 2004, pp. 1671-1685.

${ }^{5}$ Zerefos, C.S., Eleftheratos, K., Balis, D. S., Zanis, P., Tselouidis, G., and Meleti, C., “ Evidence of impact on cirrus cloud formation," Atmos. Chem. Phys. Vol. 3, 2003, pp. 1633-1644.

${ }^{6}$ Stordal, F., Myhre, G., Stordal, E. J. G., Rossow, W. B., Lee, D. S., Arlander, W., and Svendby, T., "Is there a trend in cirrus cloud cover due to aircraft traffic?," Atmos. Chem. Phys. Vol. 5, 2005, pp. 2155-2162.7

${ }^{7}$ Wuebbles et al., "A Report of Findings and Recommendations of the Workshop on the Impacts of Aviation on Climate Change, Boston, MA, June 7-9, 2006," Joint Planning and Development Office Environmental Integrated Product Team, August 31, 2006, 63 pp.

${ }^{8}$ Bakan, S., Betancour, M., Gayler, V., and Grassl, H., "Contrail frequency over Europe from NOAA-satellite images," Ann. Geophysicae, Vol. 12, 1994, pp. 962-968.

${ }^{9}$ DeGrand, J. Q., Carleton, A. M., Travis, D. J., and Lamb, P. J., “A satellite-based climatic description of jet aircraft contrails and associations with atmospheric conditions, 1977-79," J. Appl. Meteorol. Vol. 39, 2000, pp. 1434-1459.

${ }^{10}$ Lee, T. F., "Jet contrail identification using the AVHRR split window," J. Appl. Meteorol., Vol. 28, No. 9, 1989, pp. 993995.

${ }^{11}$ Mannstein, H., Meyer, R., and Wendling, P., "Operational detection of contrails from NOAA-AVHRR data," Int. J. Remote Sensing, Vol. 20, 1999, pp. 1641-1660.

${ }^{12}$ Meyer, R., Mannstein, H., Meerkötter, R., Schumann, U., and Wendling, P., "Regional radiative forcing by line-shaped contrails derived from satellite data," J. Geophys. Res., Vol. 107, 2002, DOI: 10.1029/2001JD000426.

${ }^{13}$ Palikonda, R., Minnis, P., Duda, D. P., and Mannstein, H., "Contrail coverage derived from 2001 AVHRR data over the continental United States of America and surrounding areas,” Meteorol. Z., Vol. 14, 2005, pp. 525-536.

${ }^{14}$ Minnis, P., Palikonda, R., Walter, B. J., Ayers, J. K., and Mannstein, H., "Contrail properties over the eastern North Pacific from AVHRR data," Meteorol. Z., Vol. 14, 2005, pp. 515-523.

${ }^{15}$ Meyer, R., Büll, R., C. Leiter, C., Mannstein, H., S. Pechtl, S., T. Oki, T., and P. Wendling, P., "Contrail observations over southern and eastern Asia in NOAA/AVHRR data and comparisons to contrail simulations in a GCM," Intl. J. Remote Sens., Vol. 28, 2007, pp. 2049-2069.

${ }^{16}$ Mannstein, H., A. Brömser, and Bugliaro, L., "Ground-based observations for the validation of contrails and cirrus detection in satellite imagery," Atmos. Meas. Tech., Vol. 3, 2010, pp. 655-669.

${ }^{17}$ Kärcher, B., Burkhardt, U., Unterstrasser, S., and P. Minnis, P., "Factors controlling contrail cirrus optical depth," Atmos. Chem. Phys., Vol. 9, 2009, pp. 6229-6254.

${ }^{18}$ Duda, D. P., Khlopenkov, K., and Minnis, P., "Two new contrail detection methods for the compilation of a global climatology of contrail occurrence," Proc. AMS $13^{\text {th }}$ Conf. Cloud Phys., Portland, OR, June 27 - July 2, Paper P2.76, 2010 URL: http://ams.confex.com/ams/13CldPhy13AtRad/techprogram/paper_171549.htm [cited 20 June 2011].

${ }^{19}$ Minnis, P., Young, D. F., Nguyen, L., Garber, D. P., Smith, W. L., Jr., and Palikonda, R., "Transformation of contrails into cirrus during SUCCESS," Geophys. Res. Lett., Vol. 25, pp. 1157-1160.

${ }^{20}$ Carleton, A., Travis, D. J., Master, K., and Vezhapparambu, S., "Composite atmospheric environments of jet contrail outbreaks for the United States," J. Appl. Meteorol Climatol., Vol. 47, 2008, pp. 641-667.

${ }^{21}$ Minnis, P., Ayers, J. K., Nordeen, M. L., and Weaver, S. P., "Contrail frequency over the United States from surface observations," J. Climate, Vol. 16, pp. 3447-3462.

${ }^{22}$ Garber, D., Minnis, P., and Costulis, P. K., “A commercial flight track database for upper tropospheric aircraft emission studies over the USA and southern Canada," Meteorol. Z., Vol. 14, pp. 445-452.

${ }^{23}$ Riencker, M. M. et al., "MERRA - NASA's Modern-Era Retrospective Analysis for Research and Application," J. Climate, 2011, doi: 10:1175/JCLI-D-11-000015.1, in press.

${ }^{24}$ Minnis, P., et al., 'Cloud detection in non-polar regions for CERES using TRMM VIRS and Terra and Aqua MODIS data,' IEEE Trans. Geosci. Remote Sens., Vol. 46, 2008, pp. 3857-3884.

${ }^{25}$ Minnis, P., et al., "CERES Edition-2 cloud property retrievals using TRMM VIRS and Terra and Aqua MODIS data, Part I: Algorithms,” IEEE Trans. Geosci. Remote Sens., Vol. 49, doi: 10.1109/TGRS.2011.2144601. 